\title{
Antihypertensive Therapy and Adherence to It of Patients with Hypertension and with Combination of Hypertension and Diabetes Mellitus
}

\author{
Sergeyeva Victoria, Glukhova O. E. and Magomedova H. M. \\ Department of Hospital Therapy, the Medical Faculty, Saratov State Medical University of V.I. Razumovskiy, Saratov, Russia
}

\begin{abstract}
Among patients with hypertension and with a combination of hypertension and diabetes mellitus the majority does not reach target values of blood pressure levels. There is a number of lacks in ambulatoryantihypertensive therapy and adherence to it. The considerable number of patients continues to accept therapy nonregularly, the choice of antihypertensive drugs and their combinations is not always adequate, non-medicamental actions aren't realized by all patients. At the same time the powerful negative contribution is associated with low adherence of patients to ambulatoryantihypertensive therapy.
\end{abstract}

Key words: Hypertension, antihypertensive therapy, adherence.

\section{Introduction}

The arterial hypertension (AH) is the most widespread disease all over the world, its role in development of cardio-vascular complications (CVC) determines the relevance of timely and adequate antihypertensive therapy (AT) which main objective consists in achievement of target blood pressure (BP) level and the maximum decrease in risk of development CVC and death from them. Tactics of conducting each patient select individually after cardiovascular risk rating. Thus monotherapy at start of treatment can be chosen only for patients with low or average risk [1]. Combined AT allows influencing simultaneously at the set of various elements of pathogenesis AH: activation reninangiotensin-aldosterone and sympathoadrenal systems, disturbance of endothelial and kidneys function, myocardial and vessel wall's hypertrophy.In this context combined AT has the big advantage before monotherapy and it is recommended for all patients with high and very high risk of CVC [1]. The

Corresponding author: Sergeyeva Victoria, $\mathrm{PhD}$, research fields: cardiology, therapy. E-mail: viktoriasergeeva@mail.ru. combination of diabetes mellitus (DM) and $\mathrm{AH}$ is a special clinical situation in which the risk of microand macrovascular defeats essentially increases. In this case combinations angiotensin-converting enzyme inhibitors (ACEI), angiotensin receptor blockers (ARB) with calcium channel blockers (CCB), thiazide diuretics (TD) in low doses, highly selective $\beta$ -blockers ( $\beta$-B) are most suitable.

In addition tomedicamental correction all patients with AH should follow the actions for lifestyle changes (LC). They allow decreasing the BP, to reduce requirement in antihypertensive drugs (AD) and to raise their efficiency, favorably to affect at available risk factors (RF), to carry out primary preventive of $\mathrm{AH}$ in patients with high normal level of $\mathrm{BP}$ and in patients with RF.

Adherence to treatment is degree of conformity of patient's behavior (concerning reception of preparations, observance of a diet and other measures of lifestyle changes) to the recommendations received from the doctor [2]. Taking incorrect dose of the recommended medication or during incorrect time, admissions in reception and-or rejection of treatment represent various forms of disturbance of adherence. 

Hypertension and Diabetes Mellitus

As a rule, the most frequent variant of inadequate adherence is reception of insufficient doses of medicines and admissions within 2-3 days [2]. Correlation between success in treatment of $\mathrm{AH}$ and adherence of the patient to therapy is obvious fact [3]. The probability of successful normalization the BP directly depends on a regularity of reception $\mathrm{AD}$. The relevance of this problem is confirmed by prognostic researches executed rather recently which have shown relationship between insufficient adherence to treatment and cardiovascular risk. Besides, it is necessary to consider that bad adherence to medicinal treatment, as a rule, means also bad adherence concerning non-medicamental methods of treatment that even more interferes with decrease of BP [4]. At last, insufficient adherence to the treatment, accompanied by frequent cancellation and the subsequent renewal of treatment, increases probability of the complications connected with the first dose of reception of preparations and a withdrawal syndrome [2].

The complex estimation of ambulatory AT (both non-medicamental, and medicamental) and adherences to it of various categories of patients, including in patients with combination $\mathrm{AH}$ and $\mathrm{DM}$ became the purpose of our research.

\section{Patients and Methods}

190 patients with $\mathrm{AH}$ and with combination of $\mathrm{AH}$ and DM type 2 which were hospitalized in cardiological and endocrinological departments of the regional hospital of the Saratov city are surveyed. Group of patients with AH were 90 persons (69\% women, 31\% men), with $\mathrm{AH}$ and $\mathrm{DM}-100$ persons ( $70 \%$ women, $30 \%$ men). Groups were comparable on age and sex. The average age of all surveyed patients was $57.61 \pm 0.70$ years. Dominated were patients with stages II and III of AH. The average age of onset hypertension in patients with $\mathrm{AH}$ and $\mathrm{DM}$ was significantly lower than that in the group without DM ( $44.7 \pm 0.20$ years and $47.6 \pm 0.41$ years, respectively, $p$
$<0.05)$, that testifies to earlier onset of hypertension in patients with diabetes.

Crisis course of AH prevailed in both groups $(56 \%$ of patients in group $\mathrm{AH}$ without $\mathrm{DM}$ and $60 \%$ - in group $\mathrm{AH}$ and DM). On duration of anamnesis of $\mathrm{AH}$ patients were divided into groups of up to 5 years, from 5 to 10 years and more than 10 years. In group of patients with $\mathrm{AH}$ there was a greatest quantity of patients with the experience of disease from 5 to 10 years (37\%), in group of combination $\mathrm{AH}$ and $\mathrm{DM}-$ with the experience more than 10 years - 61\%.

For a number socially - demographic indicators of our patients was dominated by married $(77 \%$ of patients in the group $\mathrm{AH}$ without $\mathrm{DM}$, and $90 \%$ in the group $\mathrm{AH}$ and $\mathrm{DM}$ ), and on an educational level patients with secondary education in the group with AH and DM (48\%); specialized secondary education $(32 \%)$ and school education $(32 \%)$ - in the group $\mathrm{AH}$ without DM. By place of residence $-38 \%$ in the group $\mathrm{AH}$ without $\mathrm{DM}$ were inhabitants of the regional center, $36 \%$ - of towns in the region; in the group with $\mathrm{AH}$ and $\mathrm{DM}-51 \%$ - the inhabitants of towns in the region. The remaining patients are represented by residents of countryside.

At the first investigation phase was carried out surveys of patients about lifestyle modification and drug actions to correct hypertension.

The questionnaire included questions on activities of lifestyle changes, about medications received by them, their dosage, duration of therapy, the addition carried out a detailed anamnesis about the features of hypertension, its course and then the laboratoryinstrumental examination of patients to identify the stage of disease and risk.

Evaluation of adherence to therapy was conducted specially developed questionnaires, including questions about the disease and its complications, character of the current therapies, including nonmedicamental correction and self-monitoring of BP. When the number of points scored 12 and above - 
the commitment was considered high, 8-11- the average, less than 8 points -low or insufficient.

Statistical processing of data produced with packages Microsoft Exel 2010, Stat Plus 2009 Professional.

\section{Results}

Non-medicamental correction of $\mathrm{AH}$ includes a number of well-known measures. According to 4th revision of National recommendations about treatment and diagnostics of $\mathrm{AH}$ it includes: normalization of body weight, quitting smoking, increasing physical activity, a complex modification of diet.

Many studies have confirmed the effect of overweight on the development of AH. Relevant for today is a complex modification of the diet with increasing consumption of plant foods in the diet increased potassium, calcium and magnesium, as well as a decrease in consumption of animal fats. The American Heart Association recommends a DASH diet. Its distinguishing feature is the combination of products such as fruits, vegetables and low fat dairy products. This food can enrich the body with potassium, calcium and magnesium, which is acting jointly; have a powerful effect on normalizing BP. In addition, modification of diet aims to reduce the level of total cholesterol in serum and thus prevent the progression of atherosclerosis.

In the group of $\mathrm{AH}$ without $\mathrm{DM}$ in $61 \%$ of identified high cholesterol, $37 \%$ excess body weight. Despite this, only $21 \%$ of patients in this group followed a diet. In the group of AH and DM - diet complied with the majority of patients (78\%), however, more than half were identified excess body weight (59\%), while $78 \%$ had hypercholesterolemia (Fig. 1). That suggests that the ongoing activities not fully effective in this patient group.

Low physical activity is one of the most common circumstances o of modern person, its increase is simple non-medicated influence on $\mathrm{AH}$ with a proven effect. Physical activity in patients was assessed by questionnaire Godin G., Sheppard R.J. In both groups of patients it's inadequate in more than half of cases (53\% in the group $\mathrm{AH}$ without $\mathrm{DM}$ and $55 \%$ in the group $\mathrm{AH}$ and $\mathrm{DM}$ ) and the correction in this direction was carried out only by $28 \%$ of patients with $\mathrm{AH}$ and $47 \%$ — with AH and DM (Fig. 1).

The role of smoking as a negative factor in the development and progression of $\mathrm{AH}$ is a proven factor. In both groups, the number of smokers approximately identical: in the group of patients with $\mathrm{AH}-19 \%$ and in the group of patients with $\mathrm{AH}$ and $\mathrm{DM}-15 \% ; 53 \%$ refused this bad habit in the group of $\mathrm{AH}$ and $47 \%$ of patients in the group of $\mathrm{AH}$ and DM (Fig. 1). In general, its low level of smoking, however, considering the prevalence of patients with high and very high risk of CVC in our investigation, there should be no smokers at all.

The estimation of drug therapy began with the start of therapy in both groups of surveyed patients. In the group of patients with AH monotherapy as the starting was appointed in $63 \%$, combined therapy in $37 \%$ of patients. In the group of - $\mathrm{AH}$ and $\mathrm{DM}-$ monotherapy - at $58 \%$, combined therapy - at $42 \%$ of patients (Fig. 3A).

A detailed study of starting monotherapy in the group of patients with $\mathrm{AH}$ without $\mathrm{DM}$ found that ACEI usage is dominated (63\%), $\beta$-AB is $-18 \%$, other groups of drugs - prescribed less often. Among them still there are such medicaments as Adelfan, clonidine. Monotherapy in the group of patients with $\mathrm{AH}$ and DM also is presented by group ACEI (75\%), $\beta$-AB accounted for $9.5 \%$ of appointments, in $8.2 \%$ outdated drugs were prescribed.

In appointing of the starting combined therapy in the group of $\mathrm{AH}$ were given preference: $\mathrm{ACEI}$ and diuretics $-27 \%$, ACEI and $\beta$-AB $-20 \%$, ACEI + $\beta-\mathrm{AB}+$ diuretic $-17 \%, \beta-\mathrm{AB}+$ diuretic $-10 \%$, the others were more rare. Combinations of drugs in the patients with $\mathrm{AH}$ and DM were represented mainly by imidazoline receptor agonists with other drugs (36\%), and ACEI with diuretics - 33\%, other combinations were less common. 

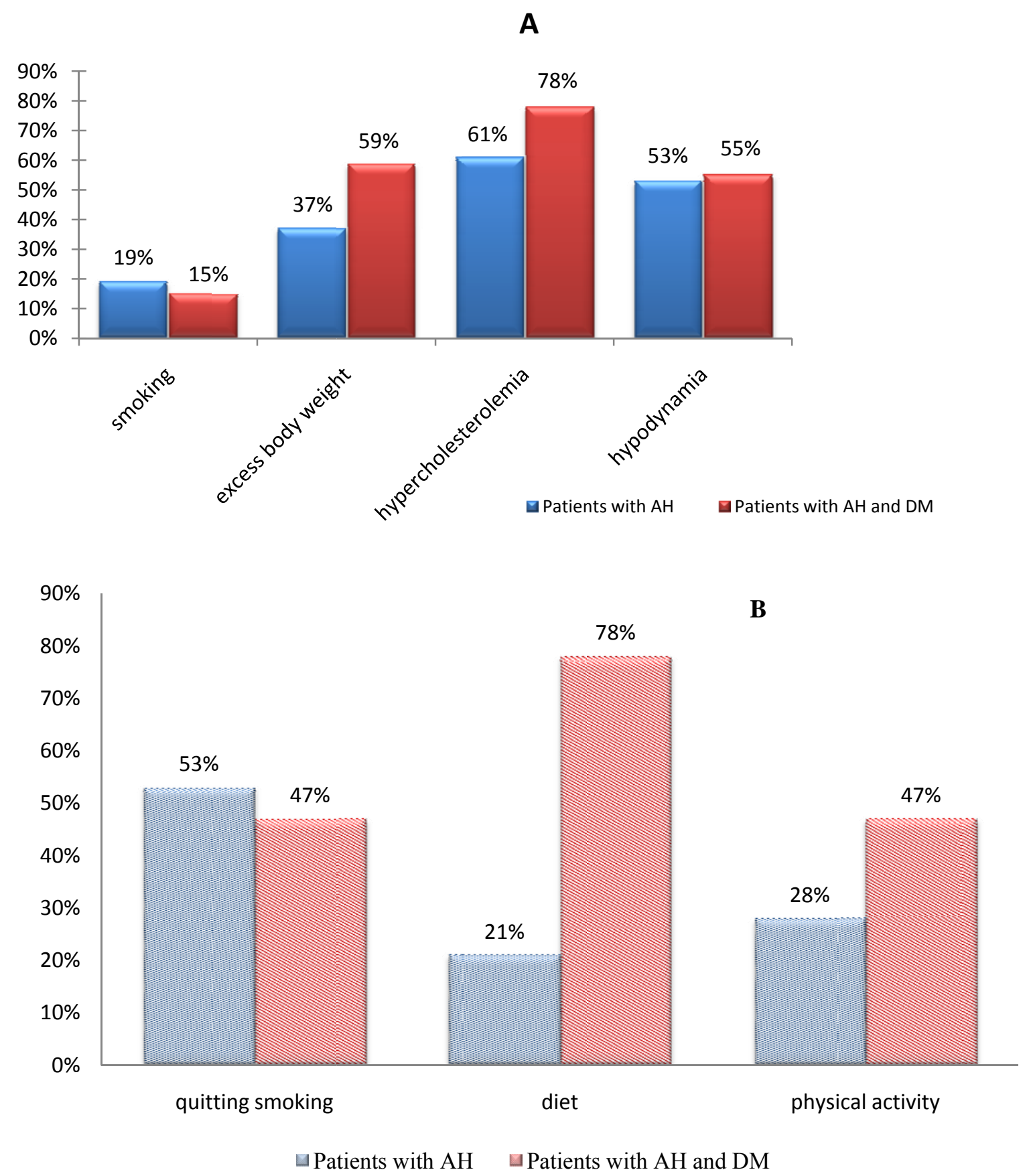

Fig. 1 Modified risk factors in groups of patients (A) and level of their correction (B).

Correction of AT ( the group of AH without DM) and taking into account the unachieved control of the disease was undertaken in $63 \%$ of cases, which was as follows: in $71 \%$ - additional medications were addedto therapy, $16 \%$ of patients transferred to long-acting form of the medications, in $7 \%$ - replaced by another groups of medications. $6 \%$ of patients required reduction of $\mathrm{AD}$ number (Fig. 2A).Correction of AT in the group with $\mathrm{AH}$ and $\mathrm{DM}$ was conducted in $75 \%$ of cases. Change of medication's groups was carried out in $45 \%$ of patients, in $44 \%$ - to primary prescription additional 
medications was added, $11 \%$ transferred to the long-acting form of the medications. In $54 \%$ of patients with $\mathrm{AH}$ without DM initially attempted to correct therapy by increased doses primary prescribed drugs. In the group with $\mathrm{AH}$ and DM, such attempts were made at $34 \%$ (Fig. 2B).

At current therapy estimation in the group of patients with $\mathrm{AH}$ despite the absence of $\mathrm{BP}$ control achieved monotherapy is maintained in $41 \%$ of patients, combined therapy is carried out in 59\%; in the group with $\mathrm{AH}$ and $\mathrm{DM}$ monotherapy is maintained in $32 \%, 68 \%$ of patients take combinations of drugs (Fig. 3B).

A detailed study of present therapy in the group of patients with AH under mono therapy as initial therapy ACEI is dominated (62\%), increased the share of $\mathrm{CCB}$ and $\beta-\mathrm{AB}$ which accounted for $8 \%$ and $20 \%$ of prescriptions, respectively. Under combined therapy: combinations of ACEI with diuretic (30\%),

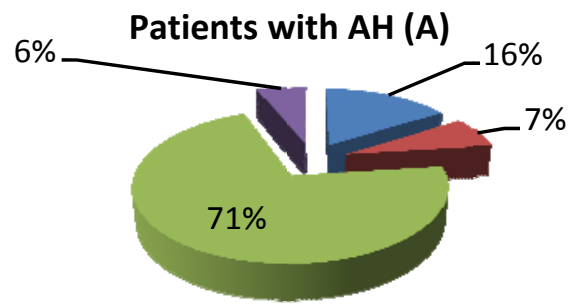

transition to the prolong AD

change to another group of $A D$

adding more AD

reduction in the number of $A D$

0

Patients with AH and DM (B)

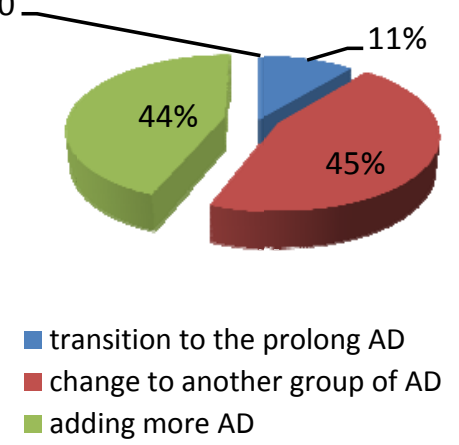

Fig. 2 Correction of Atingroups of patients.

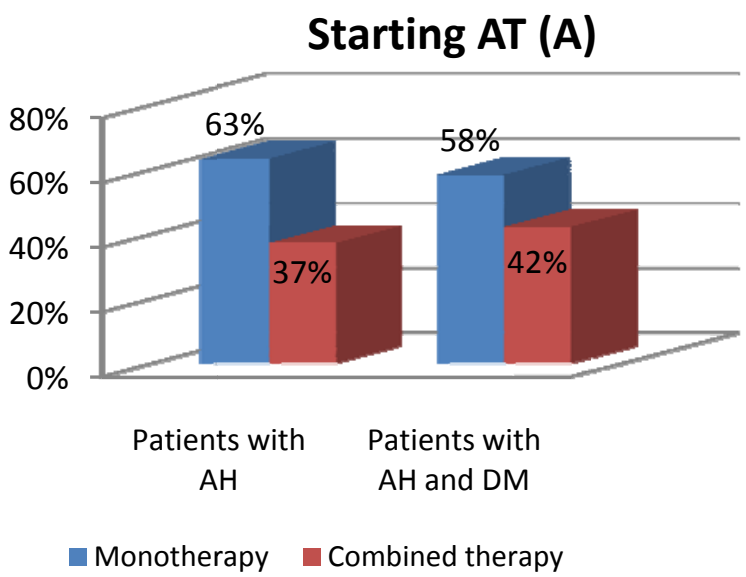

Current AT (B)

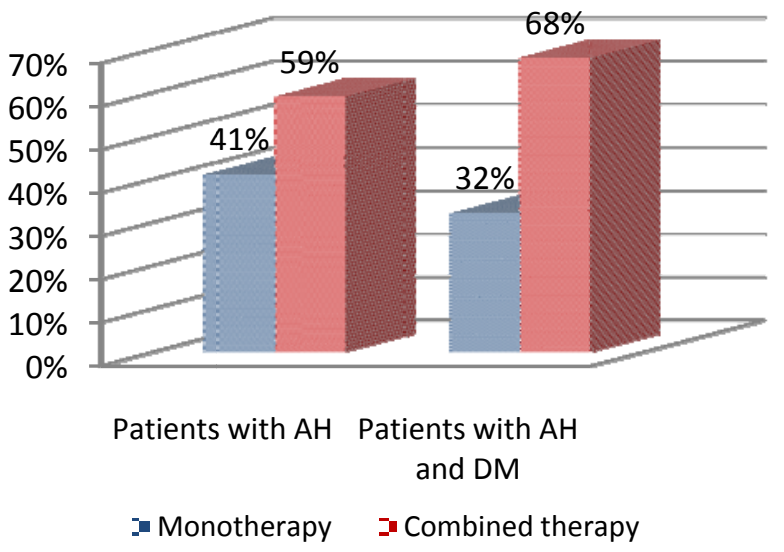

Fig. 3 Therapy estimation in the group of patients.

ACEI with $\beta-\mathrm{AB}$ (22\%), ACEI with $\beta-\mathrm{AB}$ and diuretics $(17 \%)$ are dominated. The analysis of assignments in $\mathrm{AH}$ and DM group showed that the predominant proportion of ACEI (53\%), but the number of prescriptions decreased as compared with starting therapy, but the share of CCBs increased (12\%), ARBs have appeared in the assignments (18\%). In combined therapy $30 \%$ of assignments are combinations ACEI and diuretic - as in the starting therapy, this combination is dominant, other combinations basically are presented by combination ACEI with others medications.

In $54.2 \%$ of the total number of patients failed to achieve target BP. 
As noted above, in addition to adequate medicamental AT and lifestyle modification a significant role in controlling AH plays an adherence of the patient to treatment. Adherence to treatment can be defined as "the degree to which the behavior of the patient concerning preparation reception corresponds to the accepted recommendations received from physicians". All the factors of low adherence can be divided as follows:

- features of the nature of therapy,

- factors related to physician,

- factors related to the patient,

- socio-economic factors.

The reasons for low adherence to antihypertensive therapy were studied quite extensively, they are:

- a large number of prescribed drugs,

- ineffective monitoring of blood pressure,

- high risk or the presence of side effects,

- no symptoms in increased blood pressure,

- lack of awareness of patients about the necessity of continuous constant taking AD,

- high cost of drugs.

Within the limits of an estimation of adherence to AT we have studied the regularity of AD taking by our patients. It was found that in the group with $\mathrm{AH}$ without DM irregularly take prescribed medication as nearly half of surveyed patients - 47\%, in the group with $\mathrm{AH}$ and DM 39\% adhered to nonregular mode of taking the drugs (Fig. 4). In assessing the factors influencing the nonregular reception in the group of nondiabetic hypertensive patients, $48 \%$ pointed to lack of compliance with the doctor, $18 \%$ - in good state of health during increased blood pressure, 14\% — high cost of drugs, the necessity to receive drugs several times a day indicated $11 \%$ of surveyed, side effects only $9 \%$ of patients (Fig. 5).

In the group of patients with $\mathrm{AH}$ and DM are also among the main obstacles to a constant mode of taking drugsprevails the lack of compliance with a physician (41\%), well-being during increased blood pressure in $20 \%$ of cases, side effects - in $14 \%$, necessity to

\section{Patients with AH (A)}

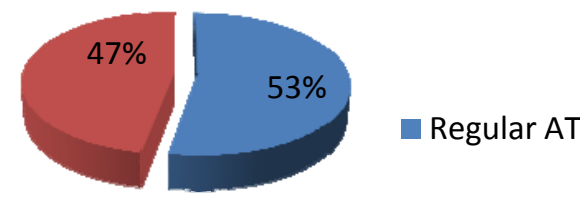

Nonregular AT

\section{Patients with AH and DM (B)}

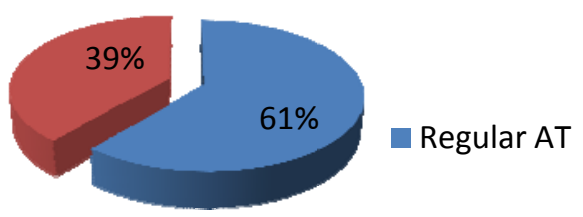

Nonregular AT

Fig. 4 Mode of Atin groups of patients.

receive the drugs a few times a day is an obstacle to keep their admission in $10 \%$ of patients, an inattention to the health — in $10 \%$, the high cost of drugs — in 5\% of the surveyed this group (Fig. 5).

In general, a high level of was found in $38 \%$ of patients in the group with $\mathrm{AH}$ and $43 \%$ — in the group of AH and DM. The average level of adherence was in $42 \%$ of the group with $\mathrm{AH}$ and in $51 \%$ of the examinees in the group of AH and DM. The remaining patients have a low adherence to AT.

There have been some regularities on adherence level in various groups of patients: it was significantly higher in women $(11.8 \pm 0.5$ points compared to men $8.9 \pm 0.6$ points, $\mathrm{p}<0.05)$, in unemployed $(12.0 \pm 0.7$ points compared with working patients $10.5 \pm 0.7, \mathrm{p}<$ $0.05)$, in inhabitants of towns of the region $(12.1 \pm 0.6$ points) compared with residents of other settlements (the inhabitants of Saratov $10.2 \pm 0.7$ points, the inhabitants of villages $-8.7 \pm 0.4$ points, $p<0.05$ ). In addition, significant differences $(p<0.05)$ in terms of adherence to therapy emerged between the groups of patients with higher education $(12.1 \pm 0.8$ points $)$ and school education $(10.1 \pm 0.1$ points). Differences in marital status in terms of adherence are not obtained. A 


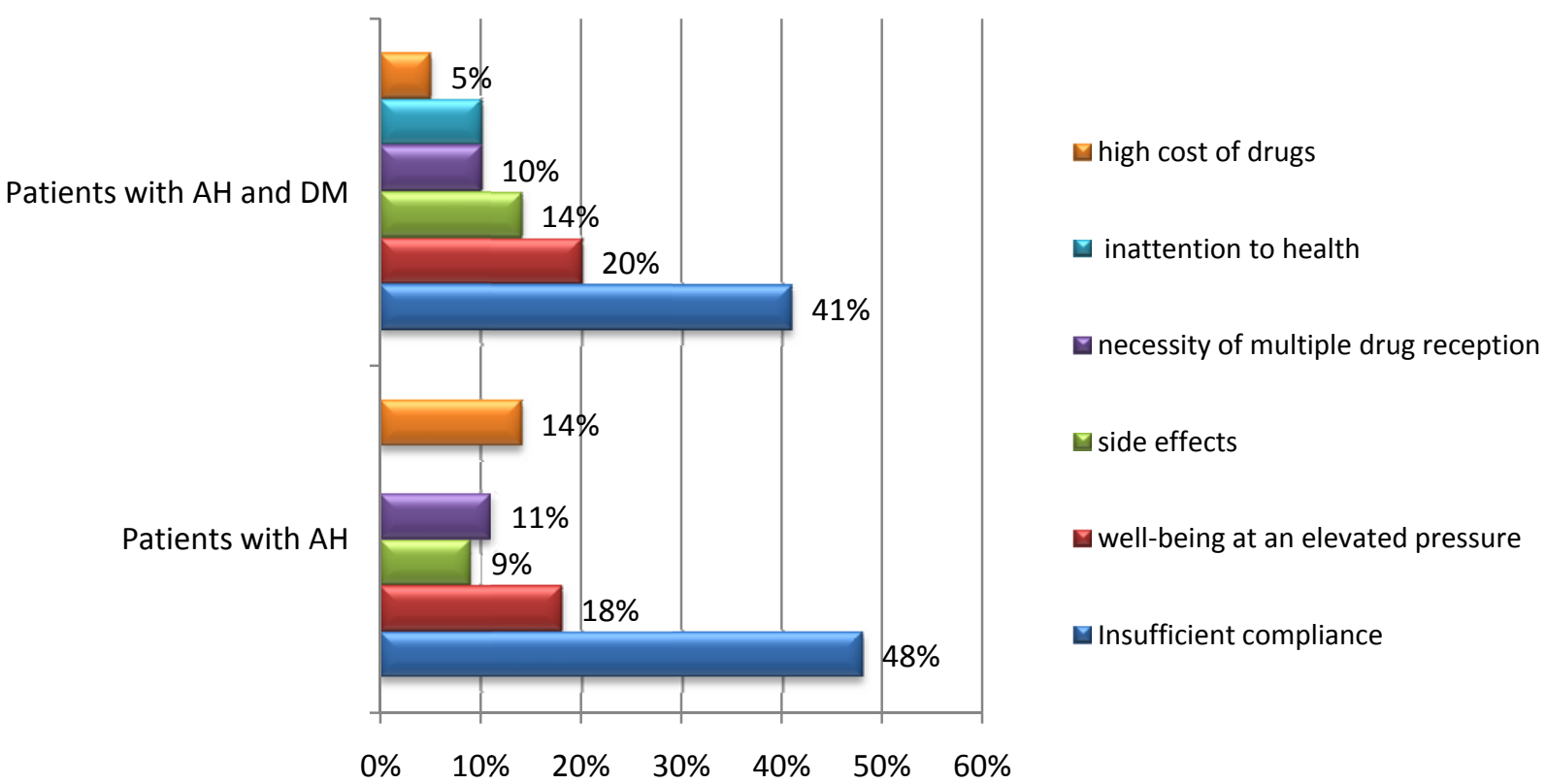

Fig. 5 Factors affecting the nonregular reception.

higher level of adherence among women may be indicative of greater attention to their health, organization, and greater confidence to physician. Inhabitants of cities in the region are also more committed to therapy than inhabitants of Saratov, which may indicate a greater employment of people in the city, lack of free time may also explain the lower adherence in the treatment of patients in the workers compared to unemployed. Educated people are more informed about their disease and in fully aware of the necessity for constant therapy.

A more detailed study of adherence in groups of patients with different stages of hypertensions showed that the lowest adherence was in stage I hypertensive patients ( $10.2 \pm 0.8$ points), it was also lower in patients with stage III of hypertension ( $10.9 \pm 0.5$ points) than in patients with hypertension with stage II $(11.5 \pm 0.9$ points), however, significant differences with the previous groups weren't obtained $(\mathrm{p}>0.05)$. It should be noted that patients with the lowest level of adherence was in the majority of the group with stage III of AH - 19\%. And patients with high level of adherence prevailed in group with II stage $\mathrm{A} \Gamma(42 \%)$.
There were significant differences $(p<0.05)$ in the adherence to AT between patients with crisis $(12.3 \pm 0.8$ points) and non-crisis course (10.16 \pm 0.4 points) of $\mathrm{AH}$.

In assessing the adherence to AT in groups depending on the lasting of $\mathrm{AH}$ obtained the following results. In the group of patients with $\mathrm{AH}$ without DM the highest found in patients with hypertension experience from 5 to 10 years ( $11.9 \pm 0.4$ points), in groups of up to 5 years and more than 10 years, rates were nearly equal $(10.5 \pm 0.4$ points and $10.3 \pm 0.1$ points, respectively, $\mathrm{p}>0.05)$. In patients with $\mathrm{AH}$ and $\mathrm{DM}$ with increasing length of hypertension the adherence rose. In the group with the experience of $\mathrm{AH}$ to 5 years its meaning was $10.3 \pm 0.1$ points, in the group with hypertension from 5 to 10 years $-11.6 \pm 0.9$ points, with the experience of more than 10 years $-13.2 \pm 0.6$ points (Fig. 6). In addition, in the group of patients with $\mathrm{AH}$ and DMrising of adherence to AT was associated with increasing length of insulinotherapy. In patients without insulinotherapy adherence to AT was $11.1 \pm 0.8$ points. In the group with insulinotherapy to 5 years, its meaning was $12.3 \pm 0.1$ points, from 5 to 10 years, $-12.9 \pm 0.4$ points, more 10 years $-14.1 \pm 0.6$ points (Fig. 7). 


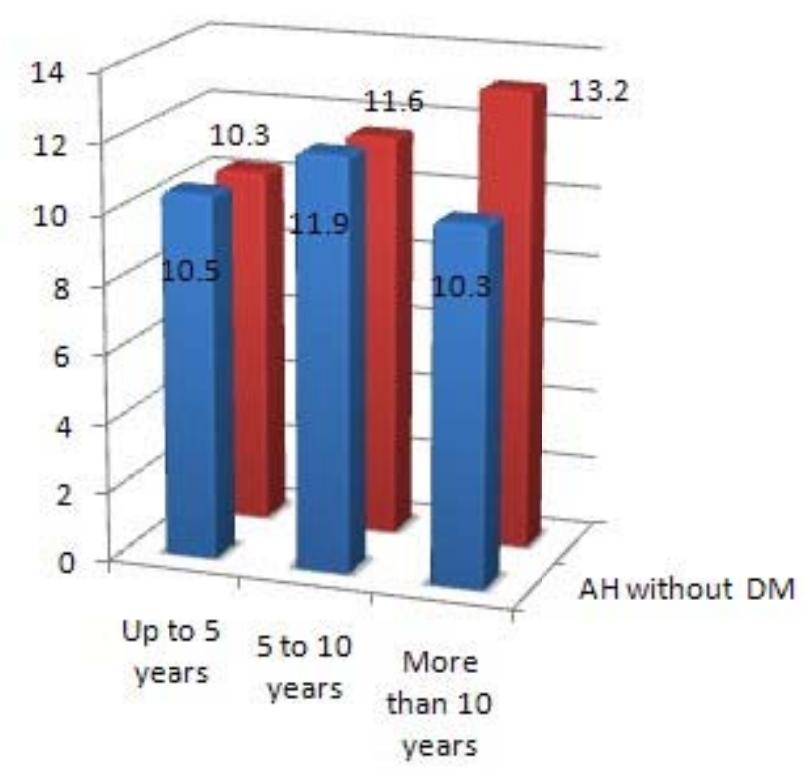

Fig. 6 Adherence to ATin groups of patients depending on the lasting of hypertension.

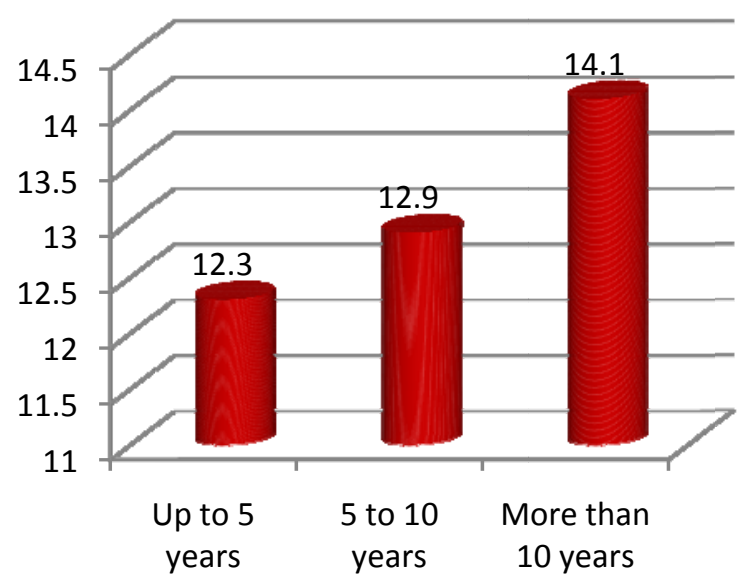

Fig. 7 Adherence to AT in the group of patients with insulinotherapy.

The last rate of adherence was one of the highest among all groups of patients.

\section{Conclusions}

The main disadvantages of antihypertensive therapy in studied patients is irrational choice of drugs, including those for the starting therapy, the use of insufficient doses and non-rational combinations of medications, and in some cases, on the contrary, polypharmacy.
Despite that the positive effect of lifestyle modification on the course of hypertension and related risk factors, as well as patient's adherence to drug therapy is a proven fact, there is not enough attention is given to non-medicamental treatments, which is possible due to lack of awareness of patients about their disease and the undoubted benefits these activities.

The adherence of antihypertensive therapy in general proved to be insufficient, and it corresponds to the middle rate. The most committed to therapy, patients with stages II and III of $\mathrm{AH}$, crisis course, experience disease from 5 to 10 years, presence of concomitant insulinotherapy in case of combination with diabetes, suggesting that patients begin to pay attention to their health with advanced pathological process with the target organ damage and associated clinical conditions, which significantly impairs health and causes more attentive to their treatment. Undoubted role is played by education of patients, the availability of free time, the material aspect, the availability of the pharmacy network.

Among the reasons of nonregular reception of preparations the most important are compliance to doctor and insufficient awareness of patient's disease and the necessity of correct its treatment, which also applies to patients who nonregularly take drugs because of the satisfactory state of health during increased blood pressure.

\section{References}

[1] Diagnostics and Treatment of Hypertension: Russian Recommendations (4th revision), 2010.

[2] M. Hill and N. Houston, Adherence to antihypertensive therapy, Chapter 131, pp. 390-392.

[3] B. Waeber, M. Burnier and H. R. Brunner, How to improve adherence with prescribed treatment in hypertensive patients? J CardiovascPharmacol 36 (2000) (suppl. 3) S23-S26.

[4] H. Horvathova, K. Kimlikova, I. Balazovjech and I. Kyselovic, Compliance and the therapeutic effect in patients with arterial hypertension, BratislLekListy 104 (4-5) (2003)149-154. 


\section{Antihypertensivetherapy and Adherence to It of Patients with Hypertension and with Combination of Hypertension and Diabetes Mellitus}

[5] T. Morozova and I. Yudina, The modern strategy for improving adherence to treatment in patients with arterialhypertension: A fixed combination of drugs, Consil Med. 12 (2010) 22-27.

[6] T. S. Lahdenpera, C. C. Wright and H. A. Kyngas, Development of a scale to assess the compliance of hypertensive patients, Int J Nurs Stud 40 (7) (2003) 677-684.

[7] M. A. Strelec and A. M. Mion, The influence of patient's consciousness regarding high blood pressure and patient's attitude in face of disease controlling medicine intake, Arc Bras Cardiol 81 (2003) 349-354.

[8] K. Port, K. Palm and M. Viigimaa, Self-reported compliance of patients receiving antihypertensive treatment: Use of a telemonitoring home care system, J TelemedTelecare 9 (2003) (Suppl. 1) S65-S66.

[9] B. Waeber, Treatment strategy to control blood pressure optimally in hypertensive patients, Blood pressure 10 (2001) 62-73.

[10] G. A. Hamilton, Measuring adherence in a hypertension clinical trial, J Manag Care Pharm 9 (5) 424-429.

[11] The major outcomes in high-risk hypertensive patients randomized to angiotensin-converting enzyme inhibitor or calcium channel blocker vs diuretic, ALLHAT, JAMA 288 (2002) 2981-2997.

[12] P. J. Murlow, Detection and control of hypertension in the population: the United States Experience, Am J Hypertens 11 (1998) 744-746.

[13] N. Col, J. E. Fanale and P. Kronholm, The role of medication non-compliance and adverse drug reactions in hospitalizations of the elderly, Arch Intern Med. 150 (1990) 841-845.

[14] T. Howard, P. Stang and E. Lydick, Increased morbidity and mortality associated with discontinuation of oral antidiabetic therapies, in: Program and Abstracts of the 35th Annual Meeting of the European Association for the Study of Diabetes, Sep. 28-Oct. 2, 1999, Brussels, Belgium.

[15] M. Pawar, Five tips for generating patient satisfaction and compliance, FamPractManag 12 (6) (2005) 44-46.

[16] D. Goleman, A. McKee and R. E. Boyatzis, Primal Leadership: Realizing the Power of Emotional Intelligence, Boston: Harvard Business School Press, 2002.
[17] C. Cuspidi, L. Lonati and L. Sampieri et al., To better know hypertension: Educational meetings for hypertensive patients, Blood Pressure 9 (2000) 255-259.

[18] A. Filippi, A. Sabatini and L. Badioli, Effects of an automated electronic reminder in changing the antiplatelet drug-prescribing behavior among Italian general practitioners in diabetic patients: an intervention trial, Diabetes Care 26 (5) (2003) 1497-1500.

[19] R. H. Frerdman, L. E. Kazis and A. Jette et al., A telecommunications system for monitoring and counseling patients with hypertension: Impact on medication adherence and blood pressure control, Am J Hypertens 9 (1996) 285-292.

[20] B. R. Haynes, D. L. Sackett and E. S. Gibson et al., Improvement of medical compliance in uncontrolled hypertension, Lancet I (1976) 1265-1268.

[21] B. Vrijens and E. Goethebeur, Comparing compliance patterns between randomized treatments, Controlled Clinical Trials 18 (1997) 187-203.

[22] B. S. Bloom, Continuation of initial antihypertensive medication after 1 year of therapy, ClinTher 20 (1998) $1-11$.

[23] P. Rudd, Clinicians and patients with hypertension: Unsettled issues about compliance, Am Heart J 130 (1995) 572-589.

[24] D. L. Sackett, R. B. Haynes and E. S. Gibson et al., Randomized clinical trial of strategies for improving medication compliance in primary hypertension, Lancet 1 (1975) 1205-1207.

[25] E. Jokasalo, H. Enlund and P. Halonen et al., Factors related to poor control of blood pressure with antihypertensive therapy, Blood Pressure 12 (2002) 22-27.

[26] J. O. Prochaska, C. A. Reddong and K. E. Evers, The transtheoretical model and stages of change, in: K. Glanz (Ed.), Health Behavior and Health Education: Theory, Research, and Practice (2nd ed.), San Francisco, Jossey-Bass, 1997.

[27] J. Benson and N. Britten, Patients decisions about whether or not to take antihypertensive drugs: Qualitative study, BMJ 325 (2002) 873. 